\title{
Editorial \\ Toward Emerging Innovations in Electrochemical Biosensing Technology
}

\author{
Tae Hyun Kim ${ }^{1,2}$ (D)
}

1 Department of Chemistry, Graduate School, Soonchunhyang University, Asan 31538, Korea; thkim@sch.ac.kr

2 Department of ICT Environmental Health System, Graduate School, Soonchunhyang University, Asan 31538, Korea

\begin{abstract}
With the progress of nanoscience and biotechnology, advanced electrochemical biosensors have been widely investigated for various application fields. Such electrochemical sensors are well suited to miniaturization and integration for portable devices and parallel processing chips. Therefore, advanced electrochemical biosensors can open a new era in health care, drug discovery, and environmental monitoring. This Special Issue serves the need to promote exploratory research and development on emerging electrochemical biosensor technologies while aiming to reflect on the current state of research in this emerging field.
\end{abstract}

Keywords: integrated biosensors; lab-on-a-chip; immunosensors; aptasensors; medical diagnostics; nanomaterials; advanced sensing platforms; environmental monitoring

The last decade has been marked by the identification of the rapidly emerging innovations in nanosystems and biotechnology [1-3]. These innovations have accelerated the creation of new electrochemical biosensors with remarkable improvements in sensitivity, selectivity, accuracy, and multiplexing capacity, along with significant size reductions [4]. Electrochemical biosensors consist of three parts: a sensitive biocomponent that recognizes the analyte, an electrochemical signal transducer or detector component that transforms the recognition into a measurable electrochemical signal, and an amplification and reader device (Figure 1). With a rich inventory of advanced material science and electronic technology such as signal processing and front-end electronic systems, researchers have overcome the limitations of conventional sensors, such as low sensitivity and lack of availability of miniaturization and integration to parallel processing chips. This Special Issue is dedicated to original results, achievements, and reviews by active researchers working on current state-of-the-art research of electrochemical biosensors. In this editorial, we intend to introduce the topic of the Special Issue, briefly describe each of the contributions that make up this Special Issue, and provide some perspectives on the future development of electrochemical biosensors.

One of the ongoing issues met by biosensing devices is the immobilization process used to intimately connect the bio-specific element onto the transducer without the loss of selectivity and sensitivity. Boris Lakard summarized the latest efforts to develop efficient immobilization approaches for biorecognition elements by entirely maintaining their biological activity, through the utilization of conducting polymers [5]. Conducting polymers have been employed in developing high-performance electrochemical biosensors owing to their advantages such as their charge transport properties and chemical versatility. In particular, conducting polymers can be easily modified and functionalized, which enables immobilization of biorecognition molecules efficiently without the inactivation of their biological properties. In his review, Boris Lakard showed the recent progress in the application of conducting polymers in the recognition of biotargets leading to the development of enzymatic biosensors, immunosensors, DNA biosensors, and whole-cell biosensors with cost-effectiveness and high sensitivity. The improvement in the sensitivity can also 
be produced by utilizing various emerging nanomaterials including 0D (quantum dots), 1D (nanowires, nanotubes), and 2D (thin films, few layers) materials. Since the discovery of graphene by Andre Geim and Konstantin Novoselov, 2D layered materials, such as graphene, black phosphorous, transition metal dichalcogenides (TMDCs), MXene, and hexagonal boron nitride, have drawn extensive attention due to their excellent properties and various application possibilities. As one of those examples, Hamami et al. reported on an electrochemical aptasensor based on molybdenum disulfide $\left(\mathrm{MoS}_{2}\right)$ nanosheets, one of TMDCs in this Special Issue [6]. They demonstrated that the proposed $\mathrm{MoS}_{2}$-based sensor exhibits rapid detection of acetamiprid insecticide with high sensitivity.

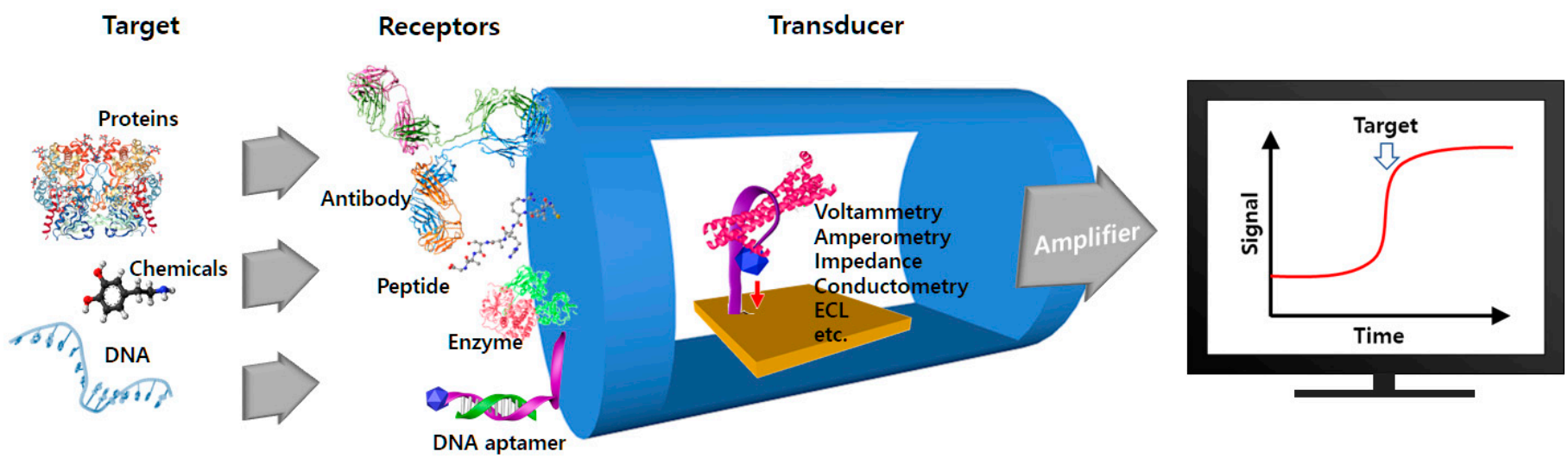

Figure 1. Illustration depicting electrochemical biosensors and their signal transduction principle.

Electrochemical sensing devices are highly suitable for miniaturization since they can be made by conventional microfabrication methods and their analytical performance is maintained even in reduced size. This enables the electrochemical devices to be implemented in various type of microsystems. In this context, two papers aimed at the utilization of electrochemical sensing techniques in microelectrode arrays and microfluidic devices. Lundblad et al. demonstrated a novel microelectrode array approach for monitoring the release of dopamine in the rat striatum [7]. The proposed approach showed highly sensitive detection of tonic (resting) and phasic release of dopamine with subsecond temporal resolution in vivo. Meanwhile, Bruijns et al. reported a microfluidic approach for DNA analysis in forensics [8]. They claimed that the microfluidic devices improve the chain of custody, reduce the contamination risk, and offer fast analysis, enabling them to be used at crime scenes. Indeed, they showed that cyclic olefin copolymer (COC)-based microfluidic device chips could be employed for real-time monitoring of DNA amplification down to $0.01 \mathrm{ng} / \mu \mathrm{L}$.

Taking advantage of their high sensitivity and selectivity, simple instrumentation, portability, outstanding compatibility, short analysis time, and low cost, advanced electrochemical biosensors have been widely used for various applications in the medical and healthcare sector, the food industry, and environmental monitoring. As a medical application of electrochemical sensors, early diagnosis of metabolic errors was introduced, and the related research was reviewed by Karastogianni et al. [9]. Their review summarized various electrochemical biosensors and point-of-care devices for the detection of branched-chain amino acids which are biomarkers for maple syrup urine disease, an inherited metabolic disorder in which the body cannot process certain amino acids properly.

To summarize, with the continuous progress of nanobiotechnology and the rapid development of electronic devices, innovative frontiers on electrochemical sensors have been launched, enabling prompt utilization of the sensors in various applications, such as medical diagnosis, drug discovery, environmental monitoring, the food industry, and light and heavy chemical industries. A forthcoming advancement in electrochemical biosensors may offer flexible and smooth integration into next-generation ICT systems, making everyday life smarter and easier. 
Acknowledgments: This work was conducted with the support of the Korea Environment Industry \& Technology Institute (KEITI), through its Ecological Imitation-based Environmental Pollution Management Technology Development Project, and funded by the Korea Ministry of Environment (MOE) (2019002800001). This work was also supported by the Soonchunhyang University Research fund.

Conflicts of Interest: The author declares no conflict of interest.

\section{References}

1. Newberry, D. Nanotechnology Past and Present: Leading to Science, Engineering, and Technology; Synthesis lectures on engineering, science, and technology; Morgan \& Claypool: San Rafael, CA, USA, 2020; ISBN 978-1-68173-861-1.

2. Webster, T.J.; Yazici, H. (Eds.) Biomedical Nanomaterials: From Design to Implementation; Healthcare technologies series; The Institution of Engineering and Technology: London, UK, 2016; ISBN 978-1-84919-964-3.

3. Salar, R.K. Biotechnology: Prospects and Applications; Springer India: New Delhi, India, 2013; ISBN 978-81-322-1683-4.

4. Zhu, C.; Yang, G.; Li, H.; Du, D.; Lin, Y. Electrochemical sensors and biosensors based on nanomaterials and nanostructures. Anal. Chem. 2015, 87, 230-249. [CrossRef] [PubMed]

5. Lakard, B. Electrochemical biosensors based on conducting polymers: A review. Appl. Sci. 2020, 10, 6614. [CrossRef]

6. Hamami, M.; Raouafi, N.; Korri-Youssoufi, H. Self-assembled MoS2/SsDNA nanostructures for the capacitive aptasensing of acetamiprid insecticide. Appl. Sci. 2021, 11, 1382. [CrossRef]

7. Lundblad, M.; Price, D.A.; Burmeister, J.J.; Quintero, J.E.; Huettl, P.; Pomerleau, F.; Zahniser, N.R.; Gerhardt, G.A. Tonic and phasic amperometric monitoring of dopamine using microelectrode arrays in rat striatum. Appl. Sci. 2020, 10, 6449. [CrossRef]

8. Bruijns, B.; Tiggelaar, R.; Gardeniers, H. A microfluidic approach for biosensing DNA within forensics. Appl. Sci. 2020, 10, 7067. [CrossRef]

9. Karastogianni, S.; Girousi, S. Electrochemical (bio)sensing of maple syrup urine disease biomarkers pointing to early diagnosis: A review. Appl. Sci. 2020, 10, 7023. [CrossRef] 